\title{
Resolution enhancement of WISDOM/ExoMars radar soundings applied on potential Martian analogs
}

\author{
Nicolas Oudart ${ }^{1}$, Valérie Ciarletti ${ }^{1}$, Alice Le Gall ${ }^{1,2}$, Marco Mastrogiuseppe ${ }^{1,3}$, Yann Hervé ${ }^{1}$, Wolf- \\ Stefan Benedix ${ }^{4}$, Dirk Plettemeier ${ }^{4}$, Vivien Tranier $^{1}$, Rafik Hassen Khodja, Christoph Statz ${ }^{4}$, and Yun \\ $\mathrm{Lu}^{4}$ \\ ${ }^{1}$ Université de Versailles Saint-Quentin, LATMOS, IMPEC, Guyancourt, France (nicolas.oudart@latmos.ipsl.fr) \\ ${ }^{2}$ Institut Universitaire de France (IUF), Paris, France \\ ${ }^{3}$ Sapienza, Rome, Italy \\ ${ }^{4}$ Tecnische Universität Dresden, Dresden, Germany
}

\begin{abstract}
The WISDOM ground penetrating radar aboard the Rosalind Franklin rover of the ExoMars 2022 mission will provide radar images of the Martian shallow subsurface down to a few meters and with a vertical resolution of a few centimetres. Such a high resolution imaging of the subsurface will be key in understanding the geological context and guiding the sample acquisition by the rover's drill. In order to enhance WISDOM resolution and meet the desired 3-cm value (the length of the samples that will be collected), a super-resolution technique known as the "Bandwidth Extrapolation" (BWE) is applied to WISDOM data. The BWE is validated on synthetic and laboratory data before being applied to observations acquired in a variety of potential Martian analog environments.
\end{abstract}

\section{Range resolution and the BWE technique}

The ExoMars 2022 rover mission is the first mission in the history of the robotic exploration of Mars able to collect $3 \mathrm{~cm}$-long samples down to $2 \mathrm{~m}$ below the surface [1]. The data collected by the WISDOM radar will be processed to produce images (radargrams) of the subsurface, that will be interpreted in terms of geological structures and provide constraints on the subsurface composition. WISDOM radargrams will bring insights into the geological context and evolution of the landing site, and eventually, guide the drilling operations [2]. The WISDOM flight model has been calibrated [3] and an improved version of the data processing pipeline is being developed.

WISDOM is a stepped-frequency radar operating at a central frequency of $1.75 \mathrm{GHz}$ over a wide frequency bandwidth $B(2.5 \mathrm{GHz})$. An Inverse Fourier Transform (IFT) is applied to the data to construct a response in time-domain. WISDOM range resolution $\square$ gis limited by the width of the synthetic pulse obtained in time domain, which is inversely proportional to the effective bandwidth of the instrument. $\square$ galso depends on the dielectric constant of the sounded medium $\square$ G Typically, for a pulse width at $6 \mathrm{~dB}$,

$$
\delta_{r}=1.21 \frac{c}{2 B \sqrt{\varepsilon_{r}}}
$$


Windowing (a technique commonly used with IFT to reduce side-lobes) further degrades the resolution, to $\sim 1.5 \square$ G for a Hamming window function. As a result, the vertical resolution of WISDOM radargrams in a subsurface with a typical dielectric constant of 4 after windowing would become $\sim 5.4 \mathrm{~cm}$ which is slightly larger than the expected $\sim 3.6 \mathrm{~cm}$ without windowing, and higher than the desired $3 \mathrm{~cm}$.

In order to improve WISDOM range resolution, we implemented a processing technique developed at the Lincoln laboratory of the MIT in 1977: the Bandwidth Extrapolation (BWE) [4]. The BWE has already been successfully applied to planetary data from radars, such as MARSIS/Mars Express [5], SHARAD/MRO [6], and the Cassini radar [7], [8]. We have adapted it for implementation in the data processing pipeline of WISDOM. The BWE technique extrapolates a radar frequency spectrum with an autoregressive linear model of the signal [9] before IFT to increase its bandwidth [10], and therefore improve its resolution in time-domain. An enhancement by a factor of 3 can be achieved [11], leading to a new range resolution better than $2 \mathrm{~cm}$ for a dielectric constant of 4 .

\section{Validation and application on potential Martian analogs}

The BWE was first validated on synthetic soundings simulating echoes from two reflectors separated by different distances with an additive white noise, to assess its ability to improve the resolution in distance while preserving the time delays and amplitudes values of the two corresponding echoes. The method was then applied to WISDOM experimental data acquired in semi-controlled environments, where the different reflectors' location is known prior to sounding. Doing so, we confirmed that the BWE leads to a significant resolution enhancement, up to a factor 3, before applying the technique to various sets of data acquired in relevant Martian analog environments. 


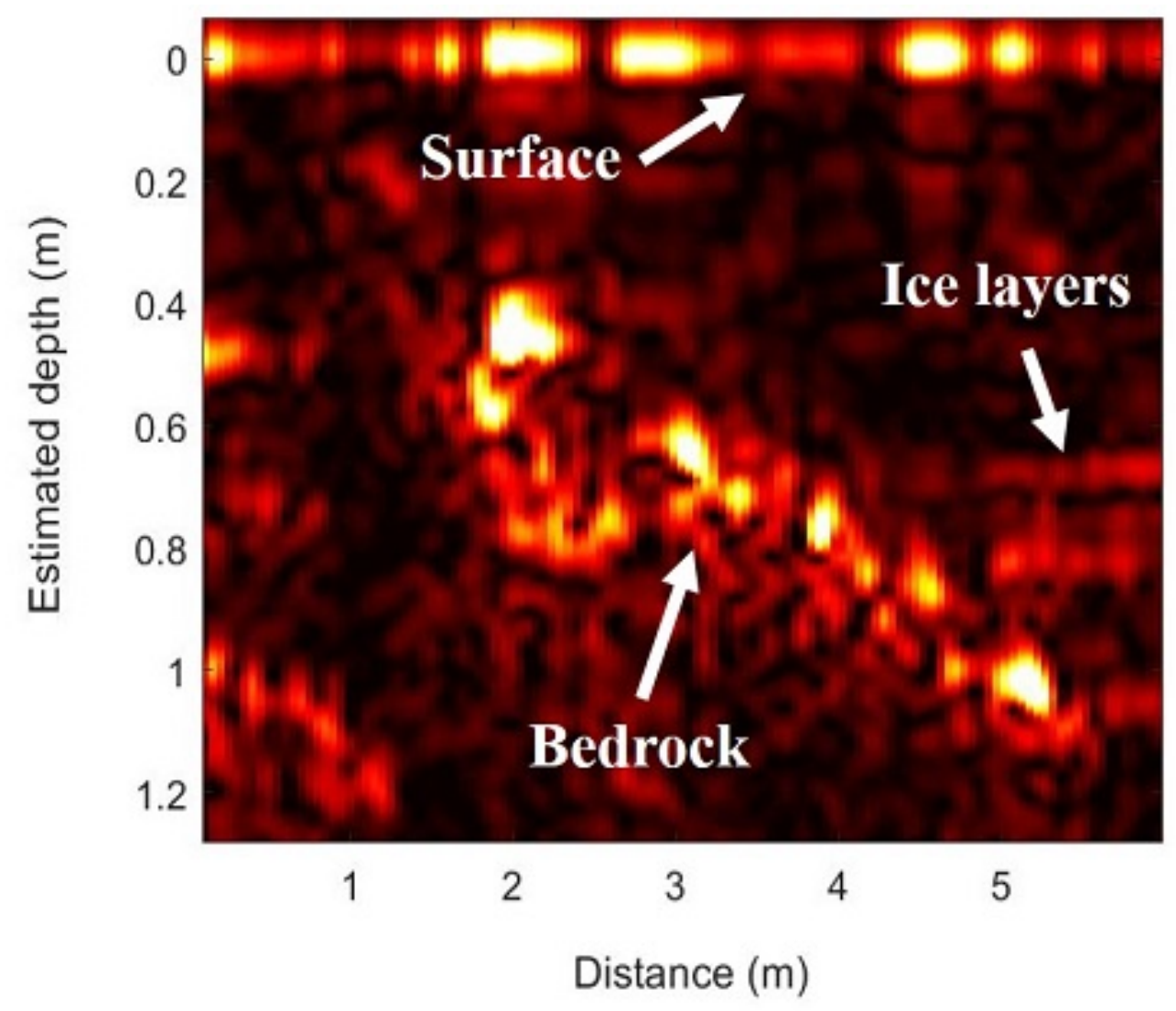

\section{Figure 1. Radargram acquired in the Dachstein Giant Ice Cave (Austria, 2012) (depth estimated with $\varepsilon_{r}=3.1$ )}

As an illustration, Figure 1 presents a radargram acquired in the Dachstein Giant Ice Cave (Austria, 2012), showing a bedrock beneath a layered ice-sheet [12], and Figure 2 the improved version after application of the BWE. The resolution is enhanced by a factor of 3 which greatly eases the detection and interpretation of buried structures. Further, new details are revealed. Other tested environments include a French Alps layered snowpack and pyroclastic deposits in the flanks of the Etna. 


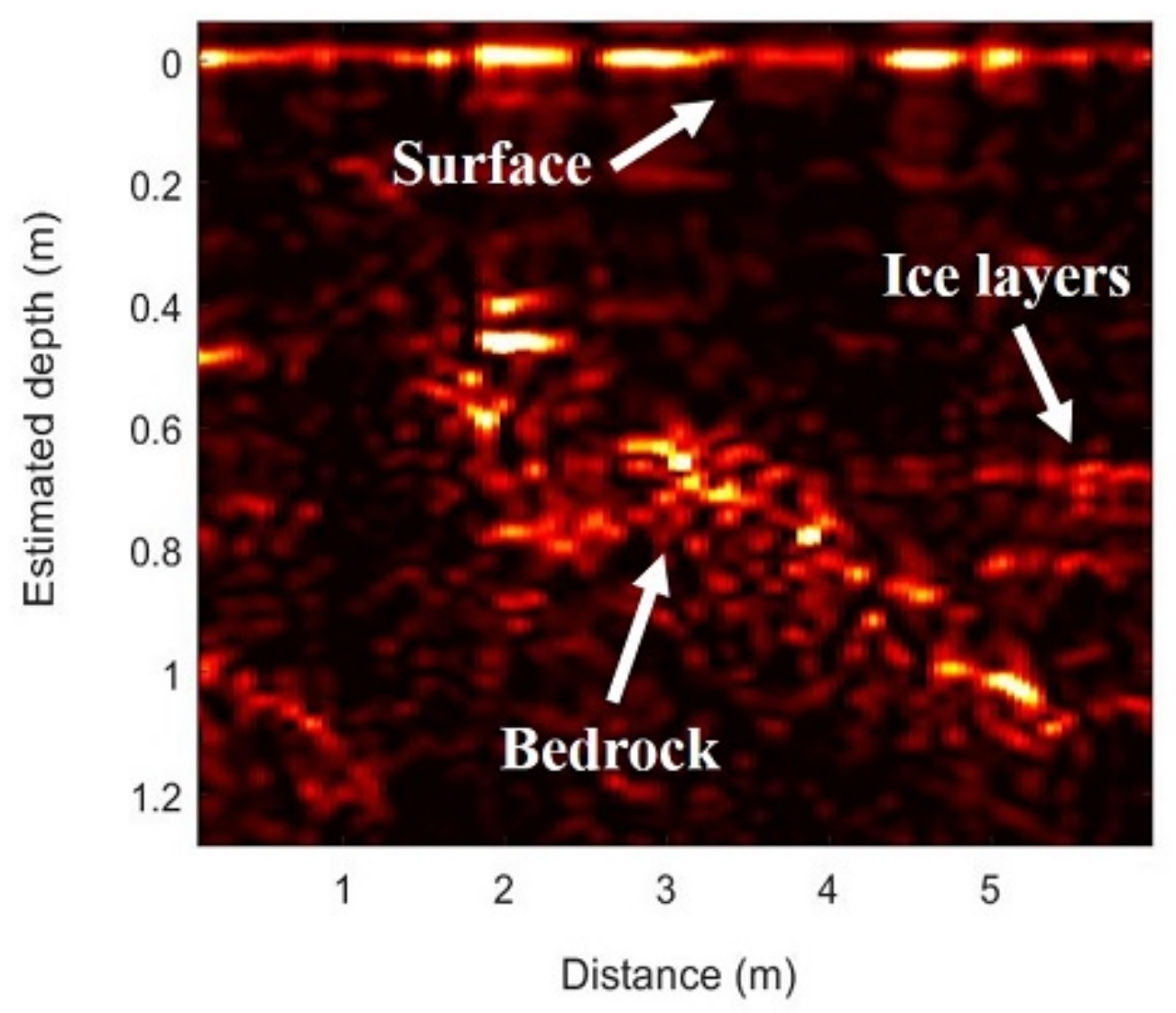

\section{Figure 2. Vertical resolution enhancement after application of the BWE to the Figure 1 radargram (depth estimated with $\varepsilon_{r}=3.1$ )}

On Mars, the BWE technique will be a powerful tool to search for structures such as layered deposits or the buried part of an outcrop, and to detect buried boulders the rover drill must avoid.

\section{Conclusion and perspectives}

The implementation of the BWE super-resolution technique proved to bring an improvement of the range resolution by a factor of 3 in WISDOM radargrams. The use of this technique will be key in the interpretation of future radargrams acquired on Oxia Planum [13], landing site of ExoMars 2022, and to accurately determine the depth to be sampled by the drill. On-going work is dedicated to adapt BWE to the full polarimetric WISDOM dataset and to propose improved algorithms for an even more accurate bandwidth extrapolation.

\section{Acknowledgments}

This research is funded by the CNES and the UVSQ.

\section{References}

[1] J. L. Vago et al., Astrobiology, 2017.

[2] V. Ciarletti et al., Astrobiology, 2017.

[3] Y. Hervé et al., Planet. Space Sci., 2020. 
[4] S. B. Bowling, MIT Lincoln Lab., 1977.

[5] M. C. Raguso et al., European Signal Processing Conference, 2018.

[6] M. C. Raguso et al., 5th IEEE International Workshop on Metrology for AeroSpace, 2018.

[7] M. Mastrogiuseppe et al., Geophys. Res. Lett., 2014.

[8] V. Poggiali et al., IEEE Trans. Geosci. Remote Sens., 2019.

[9] J. P. Burg, 37th Meet. Soc. Explor. Geophys., 1967.

[10] K. M. Cuomo, MIT Lincoln Lab., 1992.

[11] T. Moore et al., Lincoln Lab. J., 1997.

[12] S. Dorizon et al., Planet. Space Sci., 2016.

[13] C. Quantin-Nataf et al., Ninth Int. Conf. Mars 2019, 2019. 